\title{
Prospective Randomized Controlled Study to assess the Role of Dexmedetomidine on Perioperative Hemodynamics in Patients with Supratentorial Tumor undergoing Surgery
}

\author{
${ }^{1}$ Sachin Vaishnav, ${ }^{2}$ Anita Shetty, ${ }^{3}$ Manjula Sarkar
}

\begin{abstract}
The stress response to an intense painful surgical stimulus is characterized by activation of the sympathetic nervous system and an increased secretion of the stress hormones. The ability of the alpha agonist dexmedetomidine (DEX) to decrease heart rate (HR) and arterial blood pressure in perioperative period was tested. One hundred and thirty two patients undergoing craniotomy for supratentorial tumor were randomly distributed to receive either saline (B group) or DEX (A group). The placebo group received saline, whereas the treatment group (A group) received a single bolus dose of DEX $(1 \mu \mathrm{g} / \mathrm{kg})$ intravenously over 10 minutes before induction of anesthesia. Hemodynamic parameters, such as HR and arterial blood pressure were measured. Both the groups were comparable with respect to age, sex, American Society for Anesthesiologist grade, and duration of surgery. The arterial blood pressure and HR were found to be lower in the DEX group when compared with the placebo group.
\end{abstract}

Keywords: Supratentorial tumors, Dexmedetomidine, Hemodynamics.

How to cite this article: Vaishnav S, Shetty A, Sarkar M. Prospective Randomized Controlled Study to assess the Role of Dexmedetomidine on Perioperative Hemodynamics in Patients with Supratentorial Tumor undergoing Surgery. Res Inno in Anesth 2017;2(1):14-17.

Source of support: Nil

Conflict of interest: None

\section{INTRODUCTION}

The goals of neuroanesthesia are to ensure stable perioperative cerebral hemodynamics and avoid sudden rise in intracranial pressure (ICP) to prevent acute brain swelling. ${ }^{1}$ The intense surgical stimuli associated with craniotomy frequently cause sympathetic activation, which results in marked changes in systemic arterial pressure and cerebral blood flow (CBF). These cerebrovascular responses may result in elevated ICP and reduction in cerebral perfusion pressure, which can lead to cerebral

\footnotetext{
${ }^{1}$ Student (Final Year), ${ }^{2,3}$ Professor

${ }^{1-3}$ Department of Anesthesia, King Edward Memorial Hospital and Seth Gordhandas Sunderdas Medical College, Mumbai Maharashtra, India

Corresponding Author: Manjula Sarkar, Professor, Department of Anesthesia, King Edward Memorial Hospital and Seth Gordhandas Sunderdas Medical College, Mumbai, Maharashtra, India, e-mail: drmanjusarkar@gmail.com
}

ischemia, especially in patients with impaired autoregulation and compromised cerebral compliance. Thus, the prevention and control of the hemodynamic response to nociceptive stimuli are of utmost importance to preserve stable cerebral homeostasis. It is also important for neurosurgical patients for rapid and smooth recovery from anesthesia, which is often preferred to allow immediate neurological evaluation. ${ }^{1}$ The antinociceptive, sympatholytic, and anesthesia-sparing effects of $\alpha_{2}$ agonists are well documented. Dexmedetomidine (DEX) is a highly selective $\alpha_{2}$ adrenoreceptor agonist, recently introduced to anesthesia practice. ${ }^{2,3}$ It produces dose-dependent sedation, anxiolysis, and analgesia (involving spinal and supraspinal sites) without respiratory depression. It provides good intraoperative hemodynamic stability with decreased intraoperative opioid requirements. In this study, the effects of DEX infusion on hemodynamic responses to various noxious stimuli in patients undergoing craniotomy were assessed and compared with the placebo.

\section{MATERIALS AND METHODS}

After the Institutional Ethical Committee approval, this prospective randomized study was done on 132 patients undergoing elective craniotomies under general anesthesia (GA) for supratentorial intracranial space-occupying lesion. Patients between age 18 and 60 years, Glasgow coma scale 15, American Society for Anesthesiologist (ASA) grade I and II, and with no history of any drug or substance allergy were eligible. Patients on antihypertensive medication with alpha methyldopa, clonidine, or other alpha-2 adrenergic agonist, preoperative heart rate (HR) less than 45 or any heart block, pregnant women, morbid obesity, having severe hepatic and renal disease were excluded. All the 132 patients were evaluated, investigated, and written informed consent was taken. In the operating room, after establishing an intravenous (IV) line, patients were connected to monitors of electrocardiogram, HR, noninvasive blood pressure, and oxygen saturation. By using sealed envelope technique, patients were randomly allotted to one of two groups, either receiving IV DEX or IV 0.9 normal saline over a period of 10 minutes followed by IV infusion. After the completion 
of bolus drugs and 10 minutes after initiation of infusion in both the groups, patients were preoxygenated for 5 minutes and simultaneously premedicated through a separate IV line with injection Ondansetron $0.08 \mathrm{mg} / \mathrm{kg}$ IV, injection Fentanyl $2 \mu \mathrm{g} / \mathrm{kg}$ IV.

Induction was done after 3 minutes of premedication with propofol in incremental doses till the loss of eye lash reflex, then neuromuscular block was achieved with injection Rocuronium $1 \mathrm{mg} / \mathrm{kg}$. Laryngoscopy and intubation were performed with adequate size of polyvinyl chloride tube. Air entry on both sides was checked and endotracheal tube was secured properly with adhesive tape. Anesthesia was maintained with $\mathrm{N}_{2} \mathrm{O}$ and $\mathrm{O}_{2}(50+50 \%)$. For muscle relaxation, injection Rocuronium was started at the rate of $0.5 \mathrm{mg} / \mathrm{kg} /$ hour. The intraoperative depth of anesthesia was maintained with 1 minimum alveolar isoflurane concentration and adjusted as per hemodynamic status. Intraoperative end-tidal $\mathrm{CO}_{2}$ was maintained between 35 and $45 \mathrm{~mm}$ $\mathrm{Hg}$. Monitoring of HR, systolic blood pressure (SBP), diastolic blood pressure (DBP), mean atrial pressure (MAP), and $\mathrm{SPO}_{2}$ was done at preoperative, 5 minutes after bolus, 10 minutes after bolus, after induction, after laryngoscopy, at intubation, at pin insertion, at incision, followed by 15 minutes interval intraoperatively, after skin closure, at pin removal, at extubation, and finally 30 minutes interval postoperatively for 4 hours. The incidents of hemodynamic changes which are outside the predetermined window (i.e., $\pm 20 \%$ of baseline or SBP outside the range of 140 to $90 \mathrm{~mm} \mathrm{Hg}$ and $\mathrm{HR}$ outside the range of 40 to 100 per minute) were recorded and treated as follows. If SBP> $140 \mathrm{~mm} \mathrm{Hg}$, or above $20 \%$ of baseline and HR> 100 beats/minute or above $20 \%$ of baseline were treated with bolus injection Fentanyl $0.5 \mu \mathrm{g} / \mathrm{kg}$. If no desirable response was observed within 5 minutes, then second bolus injection Fentanyl $0.5 \mu \mathrm{g} / \mathrm{kg}$ was given. If no desirable response was observed within
4 minutes, then injection Esmolol was given IV $0.5 \mathrm{mg} /$ $\mathrm{kg}$ over 30 seconds increments every 5 minutes until the predetermined limits were achieved. Also, SBP $<90 \mathrm{~mm}$ $\mathrm{Hg}$ or below $20 \%$ of baseline was treated with injection Ephedrine $5 \mathrm{mg}$ IV; HR $<40$ or below 20\% of baseline was treated with injection Atropine in titrated doses. $\mathrm{N}_{2} \mathrm{O}$ administration was discontinued after skin closure. Isoflurane and Rocuronium administration was stopped after skin closure. Neuromuscular block was antagonized with injection Neostigmine $0.05 \mathrm{mg} / \mathrm{kg}$ and injection Glycopyrrolate $0.008 \mathrm{mg} / \mathrm{kg}$. Extubation was done when respiratory efforts were sufficient and patients were able to obey simple verbal commands. Then patients were shifted to recovery room and observed for 4 hours.

\section{RESULTS}

A total of 132 patients were selected for the study and were randomly divided into two groups of 66 patients each. Both the groups were comparable with respect to age, sex, ASA grade, and duration of surgery. The mean age in group I was $41.94 \pm 16.149$ (18-60) years and in group II was $41.59 \pm 13.412(18-60)$ years. The mean weight in group I was $54.97 \pm 7.284 \mathrm{~kg}$ and in group II was $57.05 \pm 6.128 \mathrm{~kg}$. In group I, there were 28 females and 38 males, whereas in group II, there were 22 females and 44 males. The sex distribution in the two groups was not statistically significant. The analysis of ASA grading was not significant and both groups were comparable.

Baseline HR between the two groups was not statistically different. After induction of anesthesia, DEX significantly decreased HR and MAP when compared with the placebo group (Graphs 1 and 2).

\section{DISCUSSION}

Perioperative hemodynamic stability is one of the most important concepts of neuroanesthesia. During

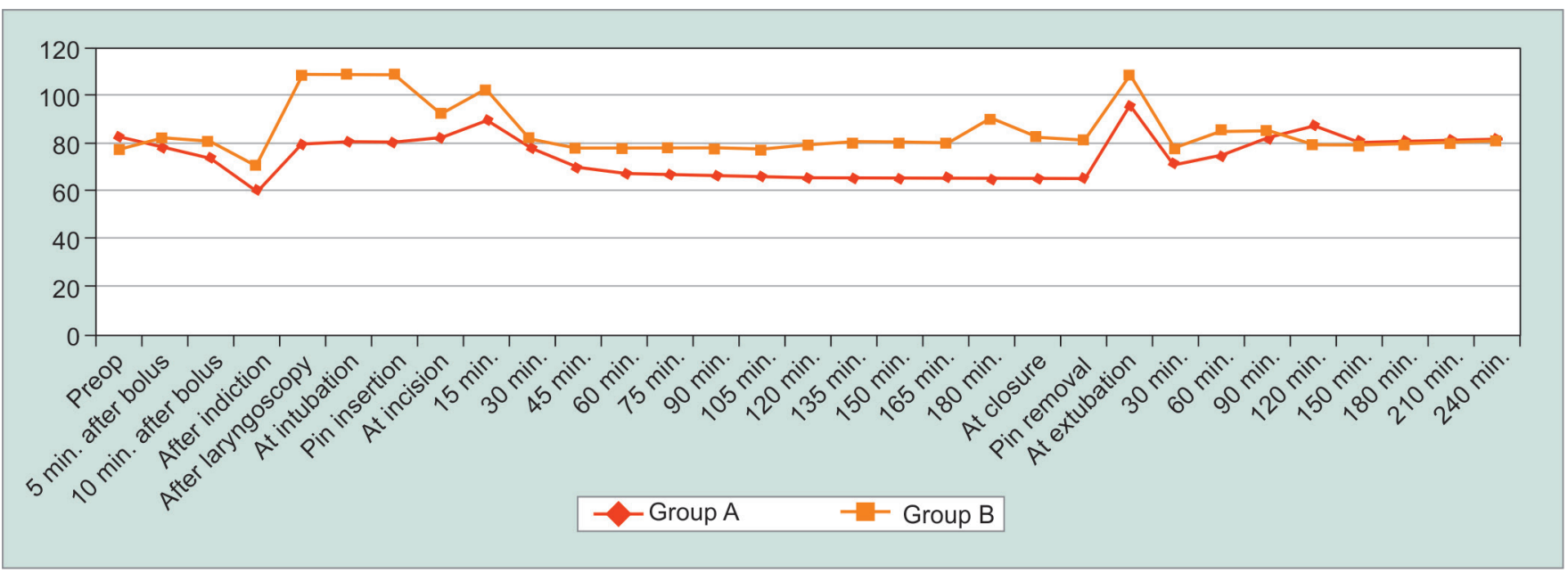

Graph 1: Comparison of heart rate in the two groups 


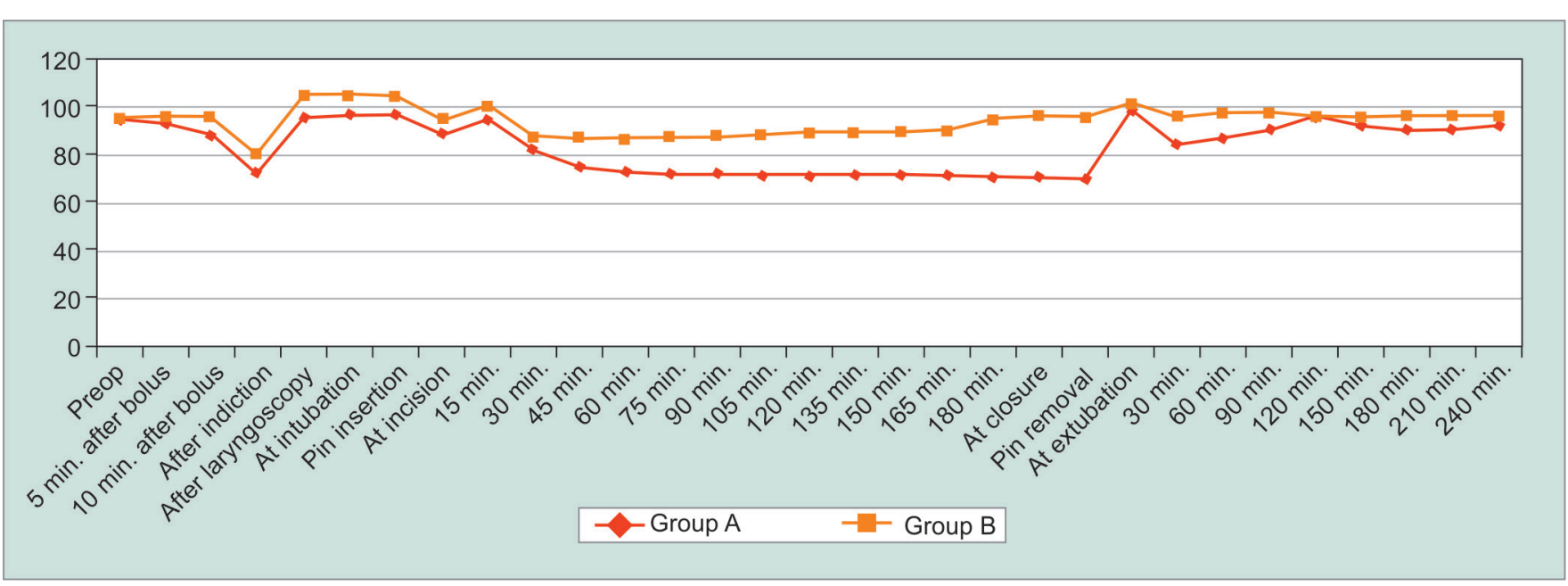

Graph 2: Comparison of mean arterial pressure in the two groups

surgery, low arterial pressure predisposes the patient to cerebral ischemia, as autoregulation of the CBF is often impaired near tumors and traumatized areas. On the contrary, abrupt rise in arterial blood pressure may cause cerebral edema or bleeding in the operating field. Hemodynamic stability is also important for rapid and smooth recovery, which is preferred for immediate neurological evaluation. $\alpha_{2}$-Adrenergic agonist could be a potentially useful anesthetic adjuvant for neurosurgical cases. ${ }^{4}$ Dexmedetomidine is a selective $\alpha_{2}$-adrenergic analgesia, and sympatholytic properties without productive significant respiratory depression. ${ }^{5}$ Its sympatholytic effect decreases MAP and HR by reducing norepinephrine release. ${ }^{6}$ It also attenuates sympathoadrenal responses to laryngoscopy and endotracheal intubation. It also provides improved hemodynamic stability during intraoperative period. It also decreases requirements for volatile anesthetics and opioids during surgery. It seems to reduce CBF secondary to cerebrovascular vasoconstriction. ${ }^{7}$ It does not increase ICP, demonstrates compatibility with neurophysiological monitoring, and may offer neuroprotection. ${ }^{8}$

In this prospective randomized study, 132 patients of either sex, age between 18 and 60 years, and ASA grade I and II undergoing elective craniotomies under GA for supratentorial intracranial space-occupying lesions were included and randomly divided into two groups: Group I (dexmedetomidine) and group II (placebo). The demographic data with respect to age, weight, sex, and ASA grade were comparable. The various hemodynamic parameters recorded were HR, SBP, DBP, and MAP. The mean HR in groups I and II was compared with each other at various time intervals, and it was observed that there was decrease in the HR in group I as compared with group II, and it was statistically significant ( $p$-value $<0.001$ ).

Talke et $\mathrm{al}^{9}$ studied the hemodynamic and adrenergic effects of perioperative DEX infusion after vascular surgery. They found that during emergence from anesthesia, HR was slower with DEX $(73 \pm 11 \mathrm{bpm})$ than with placebo $(83 \pm 20 \mathrm{bpm})(\mathrm{p}=0.006)$. The mean SBP in groups I and II was compared with each other at various time intervals, and it was observed that there was decrease in the mean SBP in group I as compared with group II and it was statistically significant ( $p$-value $<0.001)$. The mean DBP in groups I and II was compared with each other at various time intervals, and it was observed that there was decrease in the mean DBP in group I as compared with group II, and it was statistically significant (p-value $<0.001)$.

The mean MAP in groups I and II was compared with each other at various time intervals, and it was observed that there was decrease in mean MAP in group I as compared with group II, and the difference was statistically significant ( $p$-value $<0.001)$.

Tanskanen et $\mathrm{al}^{1}$ studied DEX as an anesthetic adjuvant in patients undergoing intracranial tumor surgery: They found that it blunted the tachycardic response to intubation $(p<0.01)$ and the hypertensive response to extubation $(p<0.01)$. Bekker et $\mathrm{al}^{2}$ studied the effect of DEX on perioperative hemodynamics in patients undergoing craniotomy. They concluded from this study that intraoperative infusion was effective for blunting the increases in SBP perioperatively.

\section{CONCLUSION}

From this study, it can be concluded that DEX provided intraoperative hemodynamic stability. It attenuated the hemodynamic responses to laryngoscopy, intubation, pin fixation, incision, and the emergence from anesthesia. It has significant opioid and anesthetic sparing effect. There was significantly faster recovery after extubation with DEX. There were fewer incidences of hypotension and bradycardia observed with DEX. 


\section{REFERENCES}

1. Tanskanen PE, Kyttä JV, Randell TT, Aantaa RE. Dexmedetomidine as an anaesthetic adjuvant in patients undergoing intracranial tumour surgery: a double-blind, randomized and placebo-controlled study. Br J Anaesth 2006 Nov;97(5):658-665.

2. Bekker A, Sturaitis M, Bloom M, Moric M, Golfinos J, Parker E, Babu R, Pitti A. The effect of dexmedetomidine on perioperative hemodynamics in patients undergoing craniotomy. Anesth Analg 2008 Oct;107(4):1340-1347.

3. Miller, RD. Miller's anaesthesia. 7th ed. Vol. 1. Churchill Livingstone Elsevier; 2009. Chapter 26, Intravenous anaesthetics; p. 751-756.

4. Bekker A, Sturaitis MK. Dexmedetomidine for neurological surgery. Neurosurgery 2005 Jul;57(1 Suppl):1-10.

5. Hall JE, Uhrich TD, Barney JA, Arain SR, Ebert TJ. Sedative, amnestic, and analgesic properties of small-dose dexmedetomidine infusions. Anesth Analg 2000 Mar;90(3): 699-705.

6. Guler G, Akin A, Tosun Z. Eskitascoglu E, Mizrak A, Boyaci A. Single analgesic properties of small dose DEX infusion. Anesth Analg 2000;90:699-705.

7. Ganjoo P, Farber NE, Hudetz A, Smith JJ, Samso E, Kampine JP, Schmeling WT. In vivo effects of dexmedetomidine on laser Doppler flow and pial arteriolar diameter. Anesthesiology 1998 Feb;88(2):429-439.

8. Maier C, Steinberg GK, Sun GH, Zhi GT, Maze M. Neuroprotection by the 2-adrenoceptor agonist dexmedetomidine in a focal model of cerebral ischemia. Anesthesiology 1993;79:306-312.

9. TalkeP,Chen R, Thomas B, Aggarwall A, Gottlieb A, Thorborg P, Heard S, Cheung A, Son SL, Kallio A. The hemodynamic and adrenergic effects of perioperative dexmedetomidine infusion after vascular surgery. Anesth Analg 2000 Apr;90(4):834-839. 